\title{
Non-linear Matter Spectra in Coupled Quintessence
}

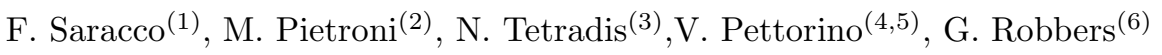

(1) INFN, Sezione di Firenze, via G. Sansone 1, Sesto Fiorentino, Firenze 50019, Italy

(2) INFN, Sezione di Padova, via Marzolo 8, Padova 35131, Italy

(3) Department of Physics, University of Athens, University Campus, Zographou 157 84, Greece

(4) Institut für Theoretische Physik, Universität Heidelberg, Philosophenweg 16, Heidelberg 69120, Germany

(5) Italian Academy for Advanced Studies in America, Columbia University, 1161 Amsterdam Avenue, New York, NY 1002\%, USA

(6) Max-Planck-Institut für Astrophysik, Karl-Schwarzschild-Strasse 1, Garching bei München 85748, Germany

\begin{abstract}
We consider cosmologies in which a dark-energy scalar field interacts with cold dark matter. The growth of perturbations is followed beyond the linear level by means of the time-renormalizationgroup method, which is extended to describe a multi-component matter sector. Even in the absence of the extra interaction, a scale-dependent bias is generated as a consequence of the different initial conditions for baryons and dark matter after decoupling. The effect is enhanced significantly by the extra coupling and can be at the 2-3 percent level in the range of scales of baryonic acoustic oscillations. We compare our results with N-body simulations, finding very good agreement.

PACS numbers: 95.35.+d, 95.36.+x, 98.80.Cq
\end{abstract}




\section{Introduction}

The power spectrum of matter perturbations reflects the evolution of the Universe since the time of matter-radiation equality. For given initial conditions, determined by the primordial spectrum (usually assumed to be scale invariant), the growth of perturbations depends on the cosmological scenario. The calculation of the present matter power spectrum can constrain this scenario through the comparison of the deduced spectrum with the observed large-scale structure. A major technical difficulty in the realization of such a program is the failure of linear perturbation theory to describe present-day fluctuations with characteristic length scales below roughly $100 \mathrm{Mpc}$. At length scales below about $10 \mathrm{Mpc}$, the evolution is highly non-linear, so that only numerical N-body simulations can capture the dynamics of the formation of galaxies and clusters of galaxies. Fluctuations with length scales of around $100 \mathrm{Mpc}$ fall within the mildly non-linear regime, for which analytical methods have been developed. These scales (corresponding to wavenumbers in the $0.03-0.25 h / \mathrm{Mpc}$ range) are of particular interest, because they correspond to the sound horizon at decoupling, which can be determined by reconstructing the oscillatory behavior of the matter power spectrum due to baryonic acoustic oscillations (BAO).

The various analytical methods [1]-9] essentially amount to resummations of subsets of perturbative diagrams of arbitrarily high order, in a way analogous to the renormalization group (RG). In this work we shall follow the approach of [5], named time-RG or TRG. In the context of the RG the various observables depend on a characteristic energy scale, and evolve as this scale is varied. The TRG uses time as the flow parameter that describes the evolution of physical quantities, such as the spectrum of perturbations. The method is characterized by conceptual simplicity. It has been applied to $\Lambda \mathrm{CDM}$ and quintessence cosmologies [5], as well as models with massive neutrinos [6].

The fundamental equations in the TRG approach are the "equations of motion", i.e. the continuity, Euler and Poisson equations. From these, equations can be derived for the time evolution of correlation functions for the density and velocity fields. The various spectra are obtained through appropriate Fourier transforms of the correlation functions. The method results in a coupled infinite system of integro-differential equations for the time evolution of the spectrum, bispectrum etc. The crucial approximation, that makes a solution possible, is to neglect the effect of the higher-order correlation functions in the evolution equations of the lower-order ones. The calculations performed so far take into account the spectrum and bispectrum and set the higher-level spectra to zero.

The procedure of truncating the system of equations is commonly employed in the applications of the Wilsonian RG to field theory or statistical physics. (For a review, see [11.) The accuracy of the calculation can be determined either by enlarging the truncated system (by including the trispectrum, for example) and examining the stability of the results, or by comparing with alternative methods. The second approach is often followed, because enlarging the truncation can increase the complexity of the calculation considerably. In the case of the TRG, the agreement with results from N-body simulations for $\Lambda \mathrm{CDM}$ has been confirmed [5]. Also, a comparative analysis of several analytical methods, using N-body simulations as a reference, has been carried out in ref. [10. The study demonstrates that the TRG remains accurate at the $1-2 \%$ level over the whole BAO range at all redshifts.

In fig. 5 of ref. 10 the deviation of the TRG prediction from a reference spectrum derived through simulations for $\Lambda \mathrm{CDM}$ is depicted. At a redshift $z=1$ the deviation is at the $1 \%$ level, or smaller, over the whole depicted range $0 \leq k \leq 0.15 h / \mathrm{Mpc}$. For $z=0$ the deviation may exceed $1 \%$ for some values of $k$, but stays below $2 \%$ over the whole depicted range. Based on these findings and the analysis of [5] we estimate an accuracy of $1 \%$ for $z=1$ and $2 \%$ for $z=0$ over the range $0 \leq k \leq 0.2 h / \mathrm{Mpc}$. The accuracy of 1-loop standard perturbation theory (SPT) can be inferred from fig. 1 of ref. [10]. At $z=1$ the accuracy is at the $1 \%$ level for $0 \leq k \leq 0.1 h / \mathrm{Mpc}$, while at $z=0$ it is at the $2 \%$ level for $0 \leq k \leq 0.05 \mathrm{~h} / \mathrm{Mpc}$. We depict these ranges in figs. 36 of the current paper. The additional approximations that we make in this paper for the study of models with non-zero coupling between dark matter and dark energy induce uncertainties at the sub-percent level. We discuss this issue in detail in subsection 3.1. For this reason the level of accuracy of our results for the power spectra in the coupled case is expected to be similar to that for $\Lambda$ CDM. Its magnitude is set by the truncations in the evolution equations (the omission of the effect of the trispectrum and higher spectra), which are similar in all models.

The purpose of the present work is threefold:

1) We extend the formalism to more complicated models. We introduce two modificiations to previous 
studies: a) Within the matter sector we allow for an arbitrary number of species with independent spectra. These include baryonic matter (BM) and cold (pressureless) dark matter (CDM). One may also consider contributions from massive neutrinos etc. b) We allow for an interaction between CDM and dark energy (DE). We consider a class of quintessence models, in which there is direct coupling between CDM and the quintessence field. The form of the interaction is a generalization of the universal coupling to all species present in scalar-tensor theories in the Einstein frame. It is modelled through the dependence of the mass of the CDM particles on the quintessence field [12]-20].

2) We test the accuracy of the method in this enlarged framework by comparing with available Nbody simulations. We perform our numerical analysis for a model for which results from simulations are given in ref. [40]. In the context of coupled quintessence, the cosmological evolution can be very diverse [14, 20, 21, 22]. It is very time-consuming to study exhaustively every model through N-body simulations. Our approach provides an alternative method, which can be much faster, while retaining the necessary accuracy. It is also important to note that, while the N-body simulations are highly accurate at the length scales of galaxies and clusters of galaxies, they are less accurate in the BAO range because of the required large volumes. On the other hand, analytical methods, like ours, are more accurate in the quasi-linear regime of large length scales. The two approaches can be viewed as complementary.

3) We provide predictions for observables for which non-linear corrections can be important. As such, we study the bias between dark and baryonic matter in the BAO range for models of coupled quintessence.

The couplings between the matter sector and DE are constrained by observations. For the BMDE coupling, the bound from the Cassini spacecraft 23 limits its order of magnitude to be below $10^{-3}$. As such small couplings produce negligible effects on the power spectrum, we assume that the BM-DE coupling is exactly zero. The coupling between CDM and DE is constrained by various considerations, such as the modification of the spectrum of the cosmic microwave background (CMB) or that of the matter distribution. A common feature of the class of models we are considering is that the presence of an additional long-range force between CDM particles, induced by the DE field, modifies their clustering properties. The various observable consequences have been discussed in the literature 24]- 40]. The strength of the CDM-DE interaction is constrained through the comparison with the observed CMB and matter spectra. It has been shown that, for particular models, the CDMDE coupling must be considerably smaller than the gravitational one [38, 39. Such constraints cannot be considered generic, because the evolution of the cosmological background and the perturbations around it varies considerably from model to model. We work within the model of [40, because our main objective is to compare with the results of N-body simulations presented there. The couplings that we consider are roughly consistent with the bounds deduced in [38, 39] for a model similar to ours.

In this work we follow a novel approach for the derivation of the fundamental equations. We derive the continuity, Euler and Poisson equations on an expanding background, starting from the conservation of the energy-momentum tensor. In order to cast these equations in a form that generalizes the standard expressions on a static background, we need to impose a certain hierarchy between the density perturbations, the velocity field and the potentials. Our derivation makes it straightforward to generalize these equations in future studies in order to take into account non-zero pressure and higher-order terms. Next, we derive the system of differential equations for the spectrum and bispectrum, within a truncation that neglects higher-level spectra. We integrate these equations numerically in order to produce the non-linear spectra at low redshift and compare with the results of N-body simulations. We study in detail the difference, usually characterized as bias, between the spectra of dark and baryonic matter. We find that the CDM-DE coupling enhances significantly the bias of the decoupled case.

In the following section we derive the evolution equations for the spectra of dark and baryonic matter. The details of the derivation for the case of one massive component are given in appendix A. The generalization for an arbitrary number of massive components is presented in appendix B. The results of the numerical integration of the evolution equations are presented in section 3 . We compare them with the results of N-body simulation. We also discuss in detail the form of the bias in the $\mathrm{BAO}$ range. 


\section{Dark matter coupled to dark energy}

\subsection{Non-linear evolution equations for the perturbations}

We assume that the energy density of the Universe receives significant contributions from three components: a) standard baryonic matter (BM); b) a species of weakly interacting, massive particles, which we identify with cold dark matter (CDM); and c) a slowly varying, classical scalar field $\phi$, whose contribution to the energy density is characterized as dark energy (DE). We also consider the possibility that there is a direct coupling between the CDM particles and the scalar field. Its equation of motion takes the form

$$
\frac{1}{\sqrt{-g}} \frac{\partial}{\partial x^{\mu}}\left(\sqrt{-g} g^{\mu \nu} \frac{\partial \phi}{\partial x^{\nu}}\right)=-\frac{d U}{d \phi}+\beta(\phi)\left(T_{C D M}\right)_{\mu}^{\mu} .
$$

We normalize all dimensionful quantities, such as the scalar field, with respect to the reduced Planck mass $M=(8 \pi G)^{-1 / 2}$. The full $M$-dependence is displayed explicitly in appendix A. Our normalization here is equivalent to setting $M=1$. Equation (2.1) can be obtained if we assume that the mass $m$ of the particles has a dependence on $\phi$ [1]. Then we have $\beta(\phi)=-d \ln m(\phi) / d \phi$. In order to be consistent with the stringent observational constraints for the baryonic sector, we assume that the interaction with the DE scalar field is confined to the CDM sector. The BM has no direct coupling to $\phi$.

For the metric, we consider an ansatz of the form

$$
d s^{2}=a^{2}(\tau)\left[(1+2 \Phi(\tau, \vec{x})) d \tau^{2}-(1-2 \Phi(\tau, \vec{x})) d \vec{x} d \vec{x}\right] .
$$

We assume that the Newtonian potential $\Phi$ is weak, $\Phi \ll 1$, and that the field $\phi$ can be decomposed as

$$
\phi(\tau, \vec{x})=\bar{\phi}(\tau)+\delta \phi(\tau, \vec{x})
$$

with $\delta \phi / \bar{\phi} \ll 1$. In general, $\bar{\phi}=\mathcal{O}(1)$ in units of $M$. The magnitude of the fluctuations of $\phi$ is expected to be similar to that of the gravitational field $\Phi$. The reason is that the source for both is the dark matter density, to which they couple with comparable strength (as will be apparent in the following). Finally, the density can be decomposed as

$$
\rho(\tau, \vec{x})=\bar{\rho}(\tau)+\delta \rho(\tau, \vec{x}) .
$$

We allow for significant density fluctuations, even though our analysis is not applicable when they are much larger than the background density. Our aim is to take into account the effect of the local velocity field $\delta \vec{v}$, when this becomes significant because of large field gradients. For subhorizon perturbations with momenta $k \gg \mathcal{H}=\dot{a} / a$, the linear analysis predicts $|\delta \vec{v}| \sim(k / \mathcal{H}) \Phi \sim(\mathcal{H} / k)(\delta \rho / \bar{\rho})$. A consistent expansion scheme can be obtained if we assume that $\Phi \ll|\delta \vec{v}| \ll 1$. Including the density perturbations, our assumptions can be summarized in the hierarchy of scales: $\Phi, \delta \phi / \bar{\phi} \ll|\delta \vec{v}| \ll$ $\delta \rho / \bar{\rho} \lesssim 1$. At the linear level, we have $\delta \vec{v}^{2} \sim \Phi(\delta \rho / \bar{\rho})$. We assume that such a relation holds at the non-linear level as well, within the range of applicability of our scheme. The velocity field is driven by the spatial derivatives of the potentials $\Phi, \delta \phi / \bar{\phi}$. As we are dealing with subhorizon perturbations, it is consistent to make the additional assumption that the spatial derivatives of $\Phi, \delta \phi$ dominate over their time derivatives. The predictions of the linear analysis allow us to make a more quantitative statement. We assume that a spatial derivative acting on $\Phi, \delta \phi$ or $\delta \vec{v}$ increases the position of that quantity in the hierarchy by one level. In this sense $\vec{\nabla} \Phi$ is comparable to $\delta \vec{v}$, while $\nabla^{2} \Phi$ is comparable to $\bar{\rho}$.

With the above assumptions, one can derive the equations that describe the evolution of the Universe. For one non-relativistic species, the derivation is presented in appendix A. It is generalized to two species in appendix B. The evolution of the homogeneous background is described by

$$
\begin{aligned}
\mathcal{H}^{2} & =\frac{1}{3}\left[a^{2} \sum_{i=1,2} \bar{\rho}_{i}+\frac{1}{2} \dot{\bar{\phi}}^{2}+a^{2} U(\bar{\phi})\right] \equiv \frac{1}{3} a^{2} \rho_{t o t} \\
\dot{\bar{\rho}}_{i}+3 \mathcal{H} \bar{\rho}_{i} & =-\beta_{i} \dot{\bar{\phi}} \bar{\rho}_{i} \\
\ddot{\bar{\phi}}+2 \mathcal{H} \dot{\bar{\phi}} & =-a^{2}\left(\frac{d U}{d \phi}(\bar{\phi})-\sum_{i=1,2} \beta_{i} \bar{\rho}_{i}\right),
\end{aligned}
$$


where we have defined $\rho_{t o t} \equiv \sum_{i} \bar{\rho}_{i}+\dot{\bar{\phi}}^{2} /\left(2 a^{2}\right)+U(\bar{\phi})$. For the CDM we have a non-zero constant $\beta_{1}=\beta$, while for BM, because of the strong constraints from solar system tests of General Relativity [23], we set $\beta_{2}=0$.

We describe the perturbations in terms of the scalar-field perturbation $\delta \phi$, the Newtonian potential $\Phi$, the density perturbations $\delta \rho_{i}$ and the velocity fields $v_{i}$. We have two Poisson equations

$$
\begin{aligned}
\nabla^{2} \delta \phi & =-a^{2} \sum_{i} \beta_{i} \delta \rho_{i} \equiv-3 \sum_{i} \beta_{i} \mathcal{H}^{2} \Omega_{i} \delta_{i} \\
\nabla^{2} \Phi & =\frac{1}{2} a^{2} \sum_{i} \delta \rho_{i} \equiv \frac{3}{2} \mathcal{H}^{2} \sum_{i} \Omega_{i} \delta_{i},
\end{aligned}
$$

with $\Omega_{i}(\tau) \equiv \bar{\rho}_{i} a^{2} /\left(3 \mathcal{H}^{2}\right)$, and the continuity and Euler equations

$$
\begin{aligned}
\delta \dot{\rho}_{i}+3 \mathcal{H} \delta \rho_{i}+\vec{\nabla}\left[\left(\bar{\rho}_{i}+\delta \rho_{i}\right) \delta \vec{v}_{i}\right] & =-\beta_{i} \dot{\bar{\phi}} \delta \rho_{i} \\
\delta \dot{\vec{v}}_{i}+\left(\mathcal{H}-\beta_{i} \dot{\bar{\phi}}\right) \delta \vec{v}_{i}+\left(\delta \vec{v}_{i} \cdot \vec{\nabla}\right) \delta \vec{v}_{i} & =-\vec{\nabla} \Phi+\beta_{i} \vec{\nabla} \delta \phi .
\end{aligned}
$$

\subsection{The CDM-BM quadruplet and the power spectra}

The evolution equations are expressed in their most useful form in terms of the density contrasts $\delta_{i} \equiv \delta \rho_{i} / \bar{\rho}_{i} \lesssim 1$ and $\theta_{i}(\mathbf{k}, \tau) \equiv \vec{\nabla} \cdot \delta \vec{v}_{i}(\mathbf{k}, \tau)$. For the Fourier transformed quantities, we obtain from eq. (2.7a)

$$
\dot{\delta}_{i}(\mathbf{k}, \tau)+\theta_{i}(\mathbf{k}, \tau)+\int d^{3} \mathbf{k}_{1} d^{3} \mathbf{k}_{2} \delta_{D}\left(\mathbf{k}-\mathbf{k}_{1}-\mathbf{k}_{2}\right) \tilde{\alpha}\left(\mathbf{k}_{1}, \mathbf{k}_{2}\right) \theta_{i}\left(\mathbf{k}_{1}, \tau\right) \delta_{i}\left(\mathbf{k}_{2}, \tau\right)=0,
$$

where

$$
\tilde{\alpha}\left(\mathbf{k}_{1}, \mathbf{k}_{2}\right)=\frac{\mathbf{k}_{1} \cdot\left(\mathbf{k}_{1}+\mathbf{k}_{2}\right)}{k_{1}^{2}}
$$

Eqs. (2.7b), 2.6a), 2.6b) give

$$
\begin{aligned}
\dot{\theta}_{i}(\mathbf{k}, \tau) & +\left(\mathcal{H}-\beta_{i} \dot{\bar{\phi}}\right) \theta_{i}(\mathbf{k}, \tau)+\frac{3 \mathcal{H}^{2} \sum_{j} \Omega_{j}\left(2 \beta_{i} \beta_{j}+1\right) \delta_{j}(\mathbf{k}, \tau)}{2} \\
& +\int d^{3} \mathbf{k}_{1} d^{3} \mathbf{k}_{2} \delta_{D}\left(\mathbf{k}-\mathbf{k}_{1}-\mathbf{k}_{2}\right) \tilde{\beta}\left(\mathbf{k}_{1}, \mathbf{k}_{2}\right) \theta_{i}\left(\mathbf{k}_{1}, \tau\right) \theta_{i}\left(\mathbf{k}_{2}, \tau\right)=0
\end{aligned}
$$

where

$$
\tilde{\beta}\left(\mathbf{k}_{1}, \mathbf{k}_{2}\right)=\frac{\left(\mathbf{k}_{1}+\mathbf{k}_{2}\right)^{2} \mathbf{k}_{1} \cdot \mathbf{k}_{2}}{2 k_{1}^{2} k_{2}^{2}} .
$$

In appendix B we discuss the above equations for an arbitrary number of non-relativistic species. Here we concentrate on the case of interest, i.e. CDM coupled to $\phi$ and BM only gravitationally coupled. We define the quadruplet

$$
\left(\begin{array}{c}
\varphi_{1}(\mathbf{k}, \eta) \\
\varphi_{2}(\mathbf{k}, \eta) \\
\varphi_{3}(\mathbf{k}, \eta) \\
\varphi_{4}(\mathbf{k}, \eta)
\end{array}\right)=e^{-\eta}\left(\begin{array}{c}
\delta_{C D M}(\mathbf{k}, \eta) \\
-\frac{\theta_{C D M}(\mathbf{k}, \eta)}{\mathcal{H}} \\
\delta_{B M}(\mathbf{k}, \eta) \\
-\frac{\theta_{B M}(\mathbf{k}, \eta)}{\mathcal{H}}
\end{array}\right)
$$

where $\eta=\ln a(\tau)$. This allows us to bring eqs. (2.8), (2.10) in the form [1, 4, 5 ]

$$
\partial_{\eta} \varphi_{a}(\mathbf{k}, \eta)+\Omega_{a b} \varphi_{b}(\mathbf{k}, \eta)=e^{\eta} \gamma_{a b c}\left(\mathbf{k},-\mathbf{k}_{1},-\mathbf{k}_{2}\right) \varphi_{b}\left(\mathbf{k}_{1}, \eta\right) \varphi_{c}\left(\mathbf{k}_{2}, \eta\right) .
$$

The indices $a, b, c$ take values $1, \ldots, 4$. The values 1,2 characterize CDM density and velocity perturbations, while 3,4 refer to BM quantities. Repeated momenta are integrated over, while repeated 
indices are summed over. The functions $\gamma$, that determine effective vertices, are analogous to those employed in 4, 5. The non-zero components are

$$
\begin{aligned}
\gamma_{121}\left(\mathbf{k}, \mathbf{k}_{1}, \mathbf{k}_{2}\right) & =\frac{\tilde{\alpha}\left(\mathbf{k}_{1}, \mathbf{k}_{2}\right)}{2} \delta_{D}\left(\mathbf{k}+\mathbf{k}_{1}+\mathbf{k}_{2}\right)=\gamma_{112}\left(\mathbf{k}, \mathbf{k}_{2}, \mathbf{k}_{1}\right) \\
\gamma_{222}\left(\mathbf{k}, \mathbf{k}_{1}, \mathbf{k}_{2}\right) & =\tilde{\beta}\left(\mathbf{k}_{1}, \mathbf{k}_{2}\right) \delta_{D}\left(\mathbf{k}+\mathbf{k}_{1}+\mathbf{k}_{2}\right) \\
\gamma_{343}\left(\mathbf{k}, \mathbf{k}_{3}, \mathbf{k}_{4}\right) & =\frac{\tilde{\alpha}\left(\mathbf{k}_{3}, \mathbf{k}_{4}\right)}{2} \delta_{D}\left(\mathbf{k}+\mathbf{k}_{3}+\mathbf{k}_{4}\right)=\gamma_{334}\left(\mathbf{k}, \mathbf{k}_{4}, \mathbf{k}_{3}\right) \\
\gamma_{444}\left(\mathbf{k}, \mathbf{k}_{3}, \mathbf{k}_{4}\right) & =\tilde{\beta}\left(\mathbf{k}_{3}, \mathbf{k}_{4}\right) \delta_{D}\left(\mathbf{k}+\mathbf{k}_{3}+\mathbf{k}_{4}\right) .
\end{aligned}
$$

The $\Omega$-matrix is

$$
\Omega(\eta)=\left(\begin{array}{cccc}
1 & -1 & 0 & 0 \\
-\frac{3}{2} \Omega_{C D M}\left(2 \beta^{2}+1\right) & 2-\beta \overline{\phi^{\prime}}+\frac{\mathcal{H}^{\prime}}{\mathcal{H}} & -\frac{3}{2} \Omega_{B M} & 0 \\
0 & 0 & 1 & -1 \\
-\frac{3}{2} \Omega_{C D M} & 0 & -\frac{3}{2} \Omega_{B M} & 2+\frac{\mathcal{H}^{\prime}}{\mathcal{H}}
\end{array}\right),
$$

where a prime denotes a derivative with respect to $\eta$.

The next step is to derive evolution equations for the power spectra. The spectra, bispectra and trispectra are defined as

$$
\begin{aligned}
\left\langle\varphi_{a}(\mathbf{k}, \eta) \varphi_{b}(\mathbf{q}, \eta)\right\rangle \equiv & \delta_{D}(\mathbf{k}+\mathbf{q}) P_{a b}(\mathbf{k}, \eta) \\
\left\langle\varphi_{a}(\mathbf{k}, \eta) \varphi_{b}(\mathbf{q}, \eta) \varphi_{c}(\mathbf{p}, \eta)\right\rangle \equiv & \delta_{D}(\mathbf{k}+\mathbf{q}+\mathbf{p}) B_{a b c}(\mathbf{k}, \mathbf{q}, \mathbf{p}, \eta) \\
\left\langle\varphi_{a}(\mathbf{k}, \eta) \varphi_{b}(\mathbf{q}, \eta) \varphi_{c}(\mathbf{p}, \eta) \varphi_{d}(\mathbf{r}, \eta)\right\rangle \equiv & \delta_{D}(\mathbf{k}+\mathbf{q}) \delta_{D}(\mathbf{p}+\mathbf{r}) P_{a b}(\mathbf{k}, \eta) P_{c d}(\mathbf{p}, \eta) \\
& +\delta_{D}(\mathbf{k}+\mathbf{p}) \delta_{D}(\mathbf{q}+\mathbf{r}) P_{a c}(\mathbf{k}, \eta) P_{b d}(\mathbf{q}, \eta) \\
& +\delta_{D}(\mathbf{k}+\mathbf{r}) \delta_{D}(\mathbf{q}+\mathbf{p}) P_{a d}(\mathbf{k}, \eta) P_{b c}(\mathbf{q}, \eta) \\
& +\delta_{D}(\mathbf{k}+\mathbf{p}+\mathbf{q}+\mathbf{r}) Q_{a b c d}(\mathbf{k}, \mathbf{p}, \mathbf{q}, \mathbf{r}, \eta) .
\end{aligned}
$$

In appendix B we summarize the derivation of the evolution equations for an arbitrary number of species. The essential approximation that we have to make in order to obtain a closed system is to neglect the effect of the trispectrum on the evolution of the bispectrum. In this way we obtain

$$
\begin{aligned}
\partial_{\eta} P_{a b}(\mathbf{k}, \eta)= & -\Omega_{a c} P_{c b}(\mathbf{k}, \eta)-\Omega_{b c} P_{a c}(\mathbf{k}, \eta) \\
& +e^{\eta} \int d^{3} q\left[\gamma_{a c d}(\mathbf{k},-\mathbf{q}, \mathbf{q}-\mathbf{k}) B_{b c d}(\mathbf{k},-\mathbf{q}, \mathbf{q}-\mathbf{k})\right. \\
& \left.+\gamma_{b c d}(\mathbf{k},-\mathbf{q}, \mathbf{q}-\mathbf{k}) B_{a c d}(\mathbf{k},-\mathbf{q}, \mathbf{q}-\mathbf{k})\right] \\
\partial_{\eta} B_{a b c}(\mathbf{k},-\mathbf{q}, \mathbf{q}-\mathbf{k})= & -\Omega_{a d} B_{d b c}(\mathbf{k},-\mathbf{q}, \mathbf{q}-\mathbf{k})-\Omega_{b d} B_{a d c}(\mathbf{k},-\mathbf{q}, \mathbf{q}-\mathbf{k})-\Omega_{c d} B_{a b d}(\mathbf{k},-\mathbf{q}, \mathbf{q}-\mathbf{k}) \\
& +2 e^{\eta}\left[\gamma_{a d e}(\mathbf{k},-\mathbf{q}, \mathbf{q}-\mathbf{k}) P_{d b}(\mathbf{q}, \eta) P_{e c}(\mathbf{k}-\mathbf{q}, \eta)\right. \\
& +\gamma_{b d e}(-\mathbf{q}, \mathbf{q}-\mathbf{k}, \mathbf{k}) P_{d c}(\mathbf{k}-\mathbf{q}, \eta) P_{e a}(\mathbf{k}, \eta) \\
& \left.+\gamma_{c d e}(\mathbf{q}-\mathbf{k}, \mathbf{k},-\mathbf{q}) P_{d a}(\mathbf{k}, \eta) P_{e b}(\mathbf{q}, \eta)\right]
\end{aligned}
$$


The formal solution of the above system is given by

$$
\begin{gathered}
P_{a b}(\mathbf{k}, \eta)=\quad g_{a c}(\mathbf{k}, \eta, 0) g_{b d}(\mathbf{k}, \eta, 0) P_{c d}(\mathbf{k}, \eta=0) \\
+\int_{0}^{\eta} d \eta^{\prime} e^{\eta^{\prime}} \int d^{3} q g_{a e}\left(\mathbf{k}, \eta, \eta^{\prime}\right) g_{b f}\left(\mathbf{k}, \eta, \eta^{\prime}\right) \\
\quad \times\left[\gamma_{e c d}(\mathbf{k},-\mathbf{q}, \mathbf{q}-\mathbf{k}) B_{f c d}\left(\mathbf{k},-\mathbf{q}, \mathbf{q}-\mathbf{k} ; \eta^{\prime}\right)\right. \\
\left.\quad+\gamma_{f c d}(\mathbf{k},-\mathbf{q}, \mathbf{q}-\mathbf{k}) B_{e c d}\left(\mathbf{k},-\mathbf{q}, \mathbf{q}-\mathbf{k} ; \eta^{\prime}\right)\right], \\
B_{a b c}(\mathbf{k},-\mathbf{q}, \mathbf{q}-\mathbf{k} ; \eta)=\quad g_{a d}(\mathbf{k}, \eta, 0) g_{b e}(-\mathbf{q}, \eta, 0) g_{c f}(\mathbf{q}-\mathbf{k}, \eta, 0) B_{d e f}(\mathbf{k},-\mathbf{q}, \mathbf{q}-\mathbf{k} ; \eta=0) \\
+2 \int_{0}^{\eta} d \eta^{\prime} e^{\eta^{\prime}} g_{a d}\left(\mathbf{k}, \eta, \eta^{\prime}\right) g_{b e}\left(-\mathbf{q}, \eta, \eta^{\prime}\right) g_{c f}\left(\mathbf{q}-\mathbf{k}, \eta, \eta^{\prime}\right) \\
\times\left[\gamma_{d g h}(\mathbf{k},-\mathbf{q}, \mathbf{q}-\mathbf{k}) P_{e g}\left(\mathbf{q}, \eta^{\prime}\right) P_{f h}\left(\mathbf{q}-\mathbf{k}, \eta^{\prime}\right)\right. \\
\quad+\gamma_{e g h}(-\mathbf{q}, \mathbf{q}-\mathbf{k}, \mathbf{k}) P_{f g}\left(\mathbf{q}-\mathbf{k}, \eta^{\prime}\right) P_{d h}\left(\mathbf{k}, \eta^{\prime}\right) \\
\left.+\gamma_{f g h}(\mathbf{q}-\mathbf{k}, \mathbf{k},-\mathbf{q}) P_{d g}\left(\mathbf{k}, \eta^{\prime}\right) P_{e h}\left(\mathbf{q}, \eta^{\prime}\right)\right],
\end{gathered}
$$

where $g_{a b}\left(\mathbf{k}, \eta, \eta^{\prime}\right)$ is the linear propagator, which gives the evolution of the field at the linear level: $\varphi_{a}^{L}(\mathbf{k}, \eta)=g_{a b}\left(\mathbf{k}, \eta, \eta^{\prime}\right) \varphi_{b}^{L}\left(\mathbf{k}, \eta^{\prime}\right)$.

The solutions can be expanded in powers of the interaction vertex $\gamma_{a b c}$, in order to establish the connection with perturbation theory [5]. The lowest order, corresponding to linear theory, is obtained by setting $\gamma_{a b c}=0$. The linear spectrum $P_{a b}^{L}$ and bispectrum $B_{a b c}^{L}$ are given by the first line of each of the above equations. The $O(\gamma)$ correction for the bispectrum is obtained by inserting $P_{a b}^{L}$ in place of $P_{a b}$ in the r.h.s. of eq. (2.20). Inserting the bispectrum at this order in eq. (2.19) generates the $O(\gamma)$ and $O\left(\gamma^{2}\right)$ contributions to the power spectrum. At this order, the result for the power spectrum reproduces exactly the one-loop expression in standard perturbation theory (SPT) 42. Iterating the procedure generates the higher-order corrections. However, differences with perturbation theory arise at higher orders, because of the approximation $Q_{a b c d}=0$ that we have made in deriving the evolution equations for the power spectrum and bispectrum.

\section{Numerical analysis}

\subsection{Approximations}

The presence of two massive species (BM and CDM) complicates the structure of the equations compared to the case where they are treated as a single fluid, discussed in [5. The full system of eqs. (2.17), (2.18) contains 74 equations, namely, 10 for the power spectra and 64 for the bispectra, compared to the 11 equations of the single-matter case. An accurate calculation also requires the discretization of the $k$-space with at least 500 points. Taking into account that the bispectra depend on three external momenta, it is apparent that the necessary computing power is significant.

The system can be reduced if additional approximations are made, based on the following observations:

- The dynamical vertices of eq. (2.14) do not mix the CDM components with the BM ones. The coupling between the two type of components is entirely due to the linear part of the equations, and especially to the $\Omega_{23}$ and $\Omega_{41}$ entries of eq. (2.15), through which the 1,2 and 3,4 indices are mixed. These originate in the Poisson equation, in which the fluctuations of all the matter species contribute universally to the gravitational potential.

- The ratio of BM and CDM density perturbations is usually characterized as bias: $b=\delta_{B M} / \delta_{C D M}$. If at early times $b$ is independent of $k$, the subsequent linear evolution preserves this independence, so that $b$ is only a function of $\eta$. At the linear level, the density-velocity and velocityvelocity spectra are proportional to the density-density ones, with $k$-independent proportionality factors. These factors are appropriate powers of the linear growth functions, so that the growing modes are selected 5 .

- The effect of the CDM-DE coupling $\beta$ on the bias can be estimated analytically in the linear approximation. For $\beta=0$ the evolution equations for CDM and BM are the same, so that, for identical initial conditions, we have $P_{11}=P_{33}=P_{13}$. For $\beta \neq 0$, the growing mode solution 
of the linearized equations has the form $\varphi_{a}(\mathbf{k}, \eta)=\varphi(\mathbf{k}, \eta)[1, f(\eta), b(\eta), b(\eta) f(\eta)]$, where $f(\eta)$, $b(\eta)$ are solutions of the system

$$
\begin{aligned}
\frac{3}{2} \Omega_{C D M}\left(2 \beta^{2}+1\right)+\frac{3}{2} \Omega_{B M} b-\frac{3}{2} \frac{1}{b} \Omega_{C D M}-\frac{3}{2} \Omega_{B M}+\beta \bar{\phi}^{\prime} f & =0 \\
f^{\prime}+\frac{\mathcal{H}^{\prime}}{\mathcal{H}} f+f+f^{2}-\frac{3}{2} \frac{1}{b} \Omega_{C D M}-\frac{3}{2} \Omega_{B M} & =0
\end{aligned}
$$

with the prime denoting derivatives with respect to $\eta=\ln a(\tau)$. For $\beta=0$, we have $b=b_{0}=1$ and $f=f_{0}(\eta)$, with $f_{0}(\eta)$ the growth function of the corresponding decoupled model. The corrections for $\beta \neq 0$ are $\mathcal{O}\left(\beta^{2}\right)$. This is obvious for those arising from the first term in eq. (3.1). The last term in the same equation has a similar effect because the evolution of the field is given by eq. 2.5c). In all models in which the CDM-DE coupling affects the cosmological evolution, the two terms in the r.h.s. of eq. (2.5c) are comparable, so that $\bar{\phi}^{\prime}=\mathcal{O}(\beta)$.

- The non-linear corrections induce a $k$-dependence at low redshifts and large $k$. Again this is an $\mathcal{O}\left(\beta^{2}\right)$ effect. This can be verified in the context of the loop expansion, as the vertices are $\beta$-independent, while the propagators for CDM and BM differ by terms of $\mathcal{O}\left(\beta^{2}\right)$.

- Finally, there is a bias induced by the initial conditions for the CDM and BM spectra, which are not identical at the end of the decoupling era. The magnitude of this effect can be deduced from our subsequent analysis, and is apparent in fig. 6, in which the bias in the BAO region is depicted at a redshift $z=1.12$. The bias deviates from 1 at a level smaller than $2 \%$ for the decoupled scenario $(\beta=0)$. We have checked that at $z=0$ the effect is below $1 \%$.

- The estimates on the $\beta$-dependence of the bias are also confirmed by fig. 6 . Within the BAO range, the bias factor $b$ receives corrections of $\mathcal{O}\left(\beta^{2}\right)$ relative to the $\beta=0$ case.

These observations offer the possibility to reduce the number of evolution equations by computing only the pure CDM or BM spectra and approximating the mixed ones. In the resulting equations for the pure spectra we make the following approximations:

- In the r.h.s. of eqs. (2.17) we approximate the mixed power spectra as the geometrical averages of the pure ones, i.e.

$$
\begin{array}{ll}
P_{13}(k, \eta) \simeq \sqrt{P_{11}(k, \eta) P_{33}(k, \eta)}, & P_{14}(k, \eta) \simeq \sqrt{P_{11}(k, \eta) P_{44}(k, \eta)}, \\
P_{23}(k, \eta) \simeq \sqrt{P_{22}(k, \eta) P_{33}(k, \eta)}, & P_{24}(k, \eta) \simeq \sqrt{P_{22}(k, \eta) P_{44}(k, \eta)} .
\end{array}
$$

If the bias were $k$-independent, we would have $P_{13}=b P_{11}, P_{33}=b^{2} P_{11}$, and the first relation would be exact. The same is true for the other relations, because of the $k$-independence of the linear growth functions. The accuracy of the resulting mixed power spectra can be estimated through the $k$-dependence of the bias. As we have discussed above, this is an $\mathcal{O}\left(\beta^{2}\right)$ effect, which is smaller than $1 \%$ for the values of $\beta$ that we use $(\beta \leq 0.1)$.

- In the r.h.s. of eqs. 2.18 the mixed components of the bispectra are approximated by the corresponding pure ones, e.g.

$$
B_{113} \simeq B_{111},
$$

and so on. The direction of the approximation (whether a CDM index is turned into a BM one, or vice versa) is decided by a majority criterium: if a bispectrum has two CDM components and a BM one, it is approximated by a purely CDM bispectrum, and vice versa. The accuracy of this approximation is determined by the magnitude of the bias. The deviation of the bias from 1 receives a correction around $1-2 \%$ at low redshifts because of the different initial conditions for $\mathrm{CDM}$ and $\mathrm{BM}$, and a correction of $\mathcal{O}\left(\beta^{2}\right)$ because of the CDM-BM coupling $\beta \neq 0$.

We emphasize that this approximation affects the calculation of the power spectra only indirectly. The approximated mixed bispectra appear in the r.h.s. of eq. (2.18) along with several pure ones. This induces an error in the pure bispectra, obtained through the integration of eq. (2.18), which is significantly smaller than $1-2 \%$. The pure bispectra then affect the calculation of the power spectra by appearing in the r.h.s. of eq. (2.17). We expect the residual effect of approximations such as (3.4) on the accuracy of the computed power spectra to be below $1 \%$. 


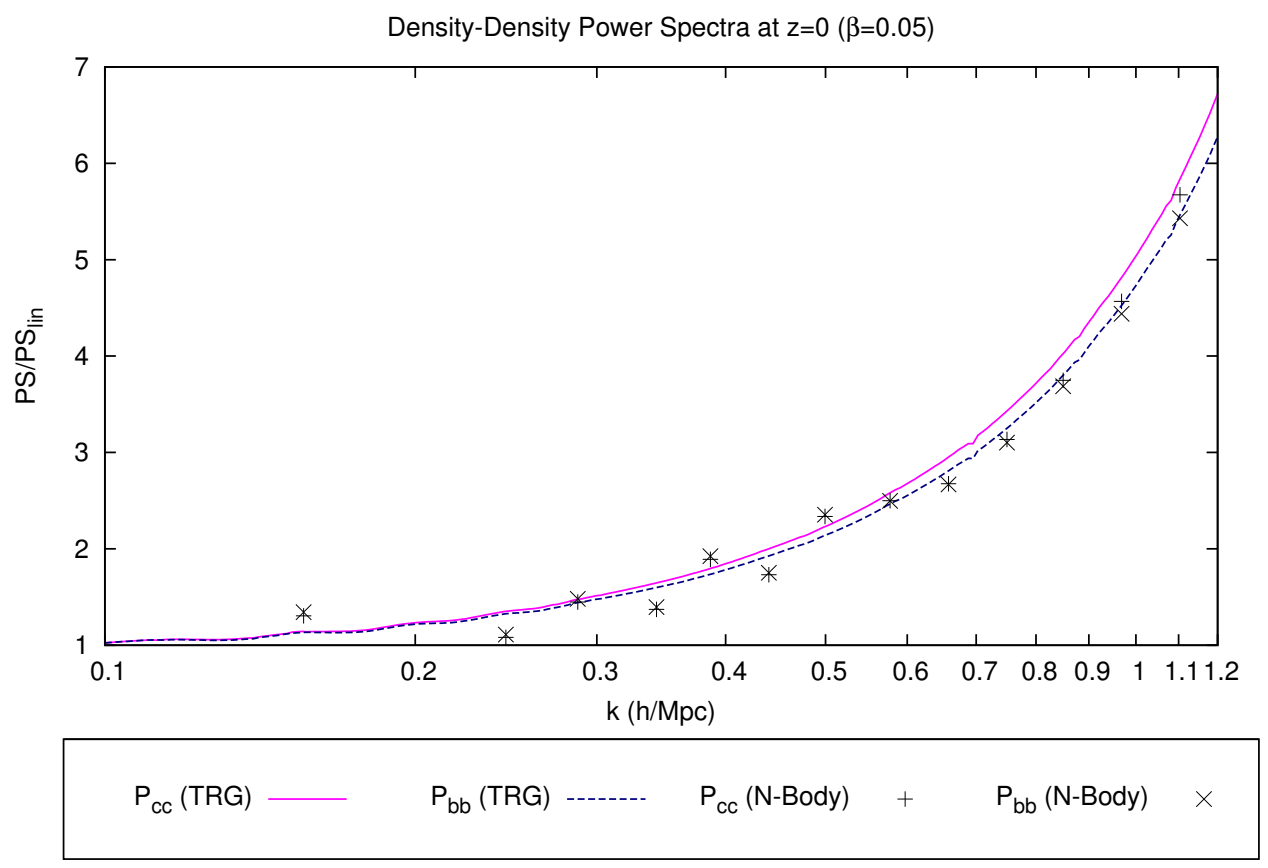

Figure 1: Comparison of results from N-body simulations and our calculation $(\beta=0.05)$. We display the ratio of the non-linear and linear spectra for $z=0$.

Through the above approximations the system is reduced to a set of 22 coupled equations, which can be solved in a way analogous to that described in Appendix B of [5]. As we have explained in detail, our approximations are expected to be valid at the sub-percent level for the power spectra within the BAO range. This can be verified a posteriori, by comparing with N-body simulations, as we will do in the following section. Of course, the most unambiguous test would be the comparison with an exact solution of the full 74 equations (2.17), (2.18). Unfortunately, it is very difficult to keep the numerical accuracy of the solution of such a huge system of integro-differential equations at the sub-percent level, in order to carry out this test.

\subsection{Results}

We have applied the formalism of the TRG to a quintessence model with non-zero coupling between $\mathrm{CDM}$ and the quintessence field $\phi$. We chose a particular model for which the matter spectrum has been calculated in [40] through numerical N-body simulations. The field has a potential $V(\phi) \sim \phi^{-\alpha}$, with $\alpha=0.143$. The present-day energy content of the Universe has $\Omega_{D E}=0.743, \Omega_{C D M}=0.213$, $\Omega_{B M}=0.044$. The Universe is assumed to have vanishing spatial curvature $\left(\Omega_{k}=0\right)$, current expansion rate $H_{0}=71.9 \mathrm{~km} \mathrm{~s}^{-1} \mathrm{Mpc}^{-1}$. The mass variance is taken $\sigma_{8}=0.769$, as calculated from the linear spectrum.

The initial conditions for the integration of the evolution equations for the spectra have been set at a redshift $z=40$. At such early times the evolution is linear to a very good approximation. We employed the implementation of the background and linear-perturbation equations in the Boltzmann code CMBEASY [4], generalized for the interacting case [40. We assumed that the primordial spectrum is scale invariant with spectral index $n=0.963$. We chose the initial value of the scalar field close to its tracker value in the uncoupled case, and adjusted the value of the dimensionful constant in its potential so as to obtain the present-day energy content listed above.

In the $\Lambda \mathrm{CDM}$ case the momentum dependence of the power spectrum ensures that the momentum integrations in the evolution equations (2.17), (2.18) are both infrared (IR) and ultraviolet (UV) finite at any order in perturbation theory. However, in order to perform numerical computations 


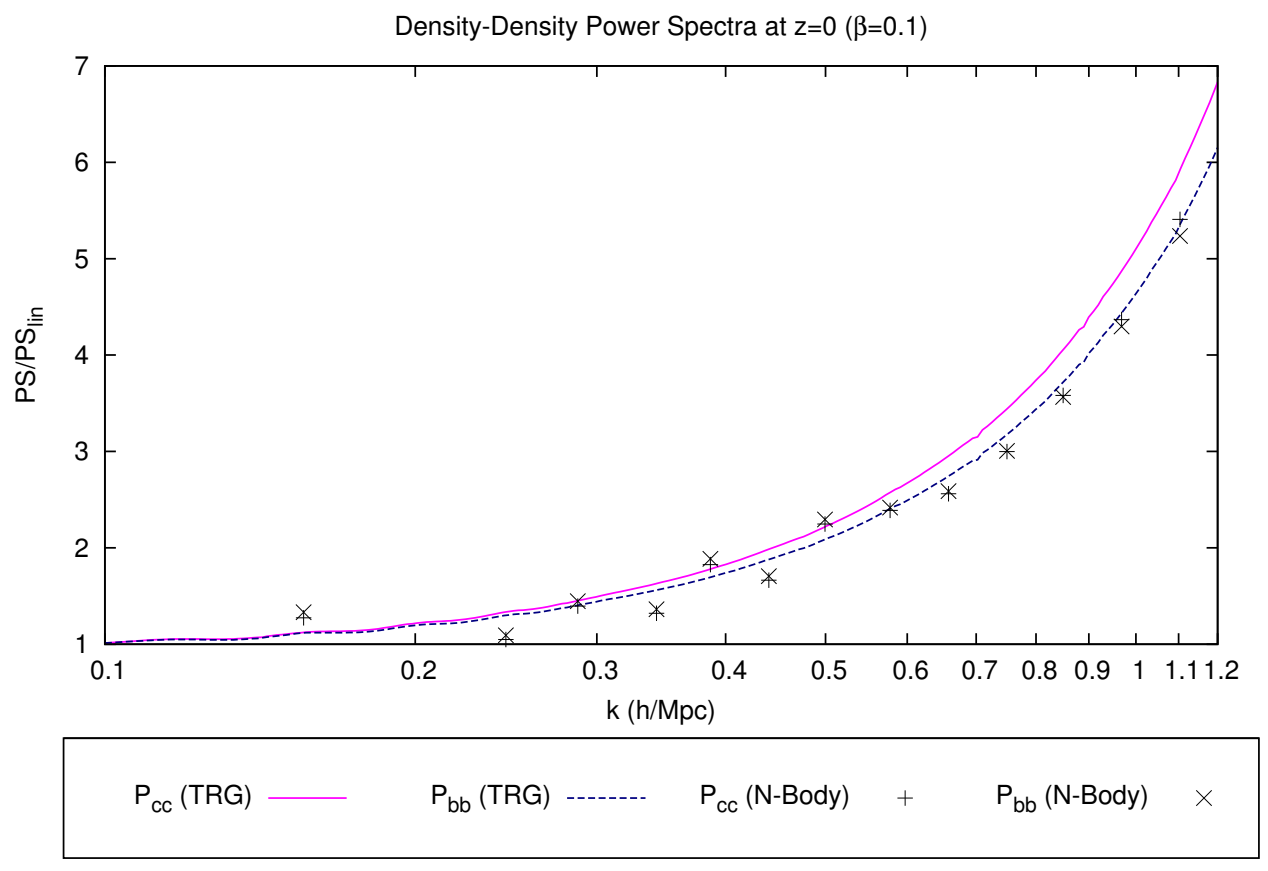

Figure 2: Comparison of results from N-body simulations and our calculation $(\beta=0.1)$. We display the ratio of the non-linear and linear spectra for $z=0$.

these integrations must be cut off both in the IR and the UV. An appropriate IR limit eliminates contributions from very large length scales, of the order of the horizon distance. In our numerical study of the case of coupled quintessence we employ an IR cutoff $k_{I R} \simeq 10^{-2} h / \mathrm{Mpc}$. We have checked that an IR cutoff $k_{I R} \simeq 10^{-3} h / \mathrm{Mpc}$ does not alter our results for the spectra. It tends, however, to increase the noise for numerical integrations with larger time steps than the ones we employ. In principle, the UV cutoff must also be chosen with care. Contributions with very large momenta correspond to length scales for which our method is not accurate. At scales below a few $\mathrm{Mpc}$ the process of virialization is crucial for the formation of galaxies and clusters of galaxies. The non-linear corrections that we take into account through the TRG are not sufficient for the quantitative description of the physics at these scales. For this reason we implement an UV cutoff $k_{U V} \simeq 2.3 \mathrm{~h} / \mathrm{Mpc}$. We have checked that the variation of $k_{U V}$ by a factor of two induces a variation of the spectra in the BAO range $(0.03 h / \mathrm{Mpc} \lesssim k \lesssim 0.25 h / \mathrm{Mpc})$ at the sub-percent level. As a result, the evolution at small length scales does not influence appreciably the evolution at the scales relevant for the BAO, which are the main focus of our calculation.

We can check the reliability of the TRG approach by comparing our results with those from $\mathrm{N}$-body simulations. In figs. 1 and 2 we display the ratio of non-linear to linear power spectra at $z=0$, for $\beta=0.05$ and 0.1 respectively. The lines correspond to our results for the CDM and BM density-density power spectra. The points indicate the results of the N-body simulations presented in [40] for the same quantities. No error bars are given for these results that would permit an accurate assessment of the level of agreement with our findings. On the other hand, we observe consistency of the two methods at scales below $0.5 \mathrm{~h} / \mathrm{Mpc}$ : The growth of the non-linear power spectrum relative to the linear one at small length scales is captured well by the TRG. It is also obvious from the same figures that the two approaches give results that start to deviate for $k \gtrsim 0.5 h / \mathrm{Mpc}$. This is expected, as the TRG cannot capture the processes of formation of bound structures that dominate at large momentum scales. Our main interest lies in the region $0.03 h / \mathrm{Mpc} \lesssim k \lesssim 0.25 h / \mathrm{Mpc}$, in which the $\mathrm{BAO}$ are visible. We also point out that the N-body simulations are rather noisy at such length scales because of finite volume effects, as they are optimized for scales smaller than the BAO range. For this reason, the two methods, N-body simulations and TRG, can be viewed as complementary: N-body 
BAO Dark Matter Density-Density Power Spectra at $z=0$ for different $\beta$
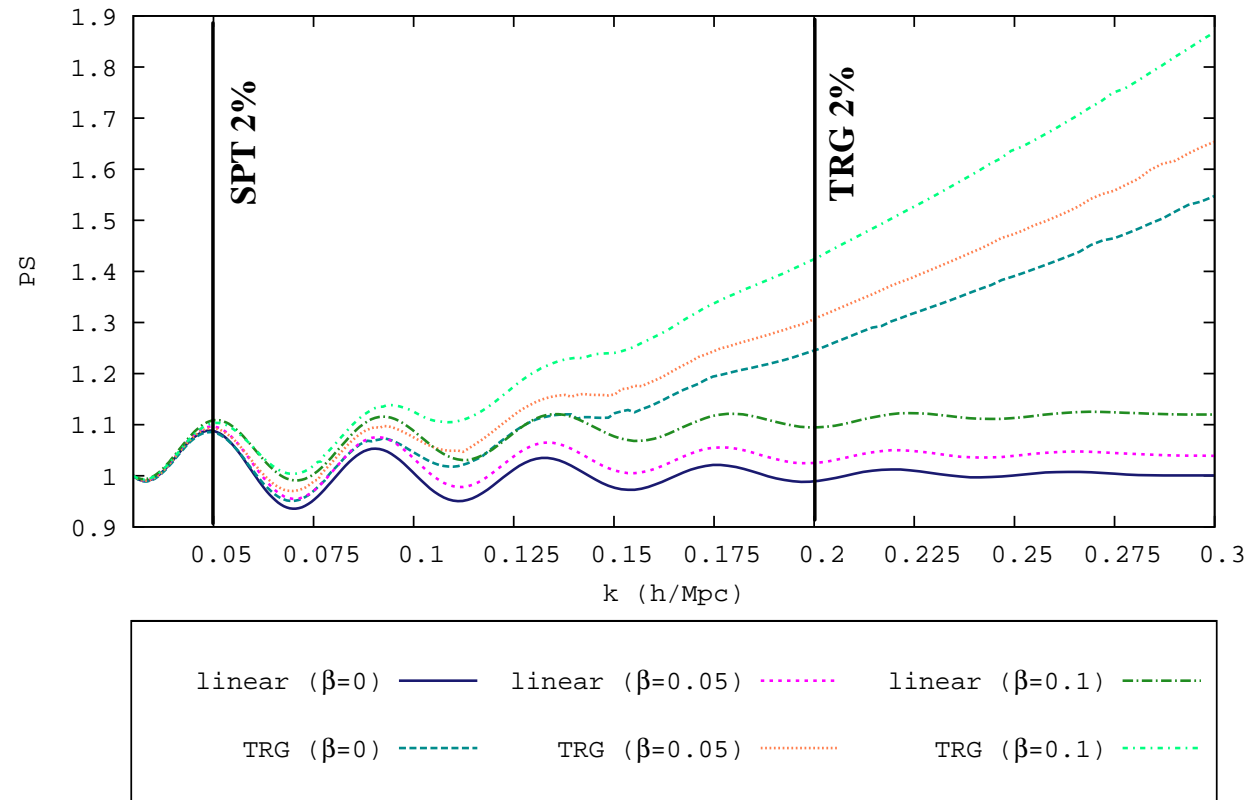

Figure 3: Dark matter density-density spectra for various $\beta$ at $z=0$, normalized with respect to the smooth function of [4]. The spectra have been multiplied by an additional $\beta$-dependent factor in order to be equal to 1 for $k \rightarrow 0$. We indicate the maximum $k$ for which a certain level of accuracy is achieved by TRG and SPT.

simulations give a reliable description of the process of virialization at relatively small length scales, while the TRG is the appropriate tool for the study of non-linear effects at the BAO range. The agreement of the two methods in the intermediate range of overlap is a confirmation their consistency.

In figs. 3, 4, 5, we display the linear and non-linear power spectra for models with $\beta=0,0.05$ and 0.1. We focus on the BAO range. In order to provide a clear depiction of the oscillatory behavior of the spectra we divide them by the smooth function of ref. [44] for $\Omega_{B M}=0$.

In fig. 3 we display the density-density power spectra for CDM for various values of $\beta$. It is apparent that the inclusion of the non-linear effects causes the spectrum to increase at large momenta. For $k \lesssim 0.06 \mathrm{~h} / \mathrm{Mpc}$ (a region that includes the first peak), the linear and non-linear spectra are indistinguishable. This indicates that the non-linear corrections become negligible at large length scales. On the other hand, the non-linear spectrum becomes significantly larger than the linear one at smaller length scales. The non-linear effects are quantitatively important in the $\mathrm{BAO}$ range. In the vicinity of the second peak they provide corrections at the level of a few percent, while in the vicinity of the third peak the corrections are about $10 \%$. These features of the non-linear corrections on the matter power spectrum in the $\beta=0$ case have been discussed in full detail in the past (for instance, in [5]).

The presence of an extra CDM-DE coupling causes an additional enhancement of the spectrum. For example, for $k=0.3 \mathrm{~h} / \mathrm{Mpc}$ the differences of the non-linear spectra for $\beta=0,0.05$ and 0.1 have roughly doubled compared to the corresponding differences of the linear spectra. A qualitative change in the spectrum is that the third peak ceases to exist for $\beta \simeq 0.1$. It is likely that the disappearance of higher-order peaks is common in models of coupled quintessence. However, it is not possible to investigate this feature in a model-independent way. The large variability of the evolution of the cosmological background and the perturbations in models of coupled quintessence makes it very difficult to identify generic features.

In fig. 4 we display the density-density power spectra of BM. They are very similar to those of CDM. A close inspection reveals that the enhancement of the spectrum with increasing $\beta$ is smaller 
BAO Baryonic Matter Density-Density Power Spectra at $z=0$ for different $\beta$
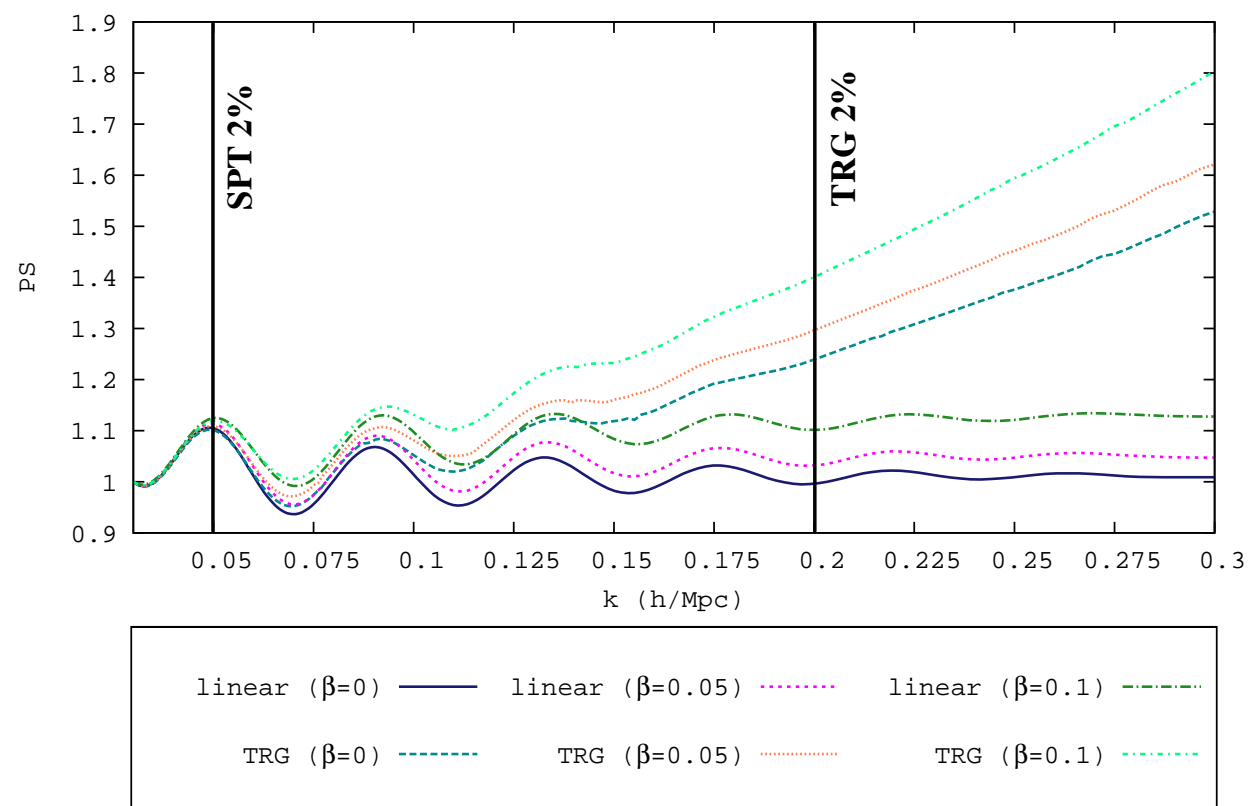

Figure 4: Baryonic matter density-density spectra for various $\beta$ at $z=0$, normalized similarly to those in fig. 3,

than for the CDM case. This is more apparent for $k=0.3 h / \mathrm{Mpc}$. The reason can be traced to the additional attractive force between CDM particles, mediated by the quintessence field. As the additional force is not felt by the baryons, the enhancement affects CDM and baryons differently, i.e. a bias is produced. This is apparent already at the linear level, but it becomes an even stronger effect at the non-linear level. The baryons are not subject to this force and tend to collapse more slowly 25. The bias is scale dependent, as we discuss in detail below.

In fig. 5 we depict the velocity-velocity power spectra for CDM. Similarly to the density-density spectra, the higher-order peaks are washed away by the combined effect of the non-linear corrections and the CDM-DE interaction. The non-linear density-density (dd), density-velocity (dv) and velocityvelocity (vv) power spectra display similar behavior. The BM spectra follow closely the variation of the corresponding DM ones. There is also a hierarchy in the magnitude of the spectra for large $k$. The reason is that the velocity field is much smaller than the density perturbation at subhorizon scales. As a result, the non-linear effects that enhance the spectra at large $k$ are more pronounced for the density-density spectra and smallest for the velocity-velocity ones.

The bias in the BAO region is depicted in fig. 6 for a redshifts $z=1.12$. It is defined as $b(k)=\left(P_{B M}(k) / P_{C D M}(k)\right)^{1 / 2}$. The small kinks in the curves give an indication of the numerical errors in our calculation. We notice that, even at vanishing coupling $(\beta=0)$, there is a bias as a result of the different initial conditions between BM and CDM after decoupling 25. However, in the $\beta=0$ case the bias parameter tends to unity at lower redshifts, as is well known. When $\beta \neq 0$ the initial unbalance between baryons and CDM is never washed out, as a consequence of the extra scalar force acting on the latter and not on the former. In linear perturbation theory, this causes a scale-independent bias in recent epochs [25]. When non-linear corrections are taken into account, the situation changes dramatically. The CDM and BM components, having different initial conditions, evolve differently even in the $\beta=0$ case, with the BM fluid being always more "linear" than the $\mathrm{CDM}$ one. Moreover, since the non-linear growth factors are scale-dependent, non-linear corrections cause a scale-dependent bias even at $\beta=0$ [26]. It is clear from fig. 6 that both effects, i.e. the bias and its scale dependence, are enhanced by a non-vanishing coupling. This feature could provide a distinctive signature for this kind of models. These results are consistent with the conclusions of refs. 
BAO Dark Matter Velocity-Velocity Power Spectra at $z=0$ for different $\beta$
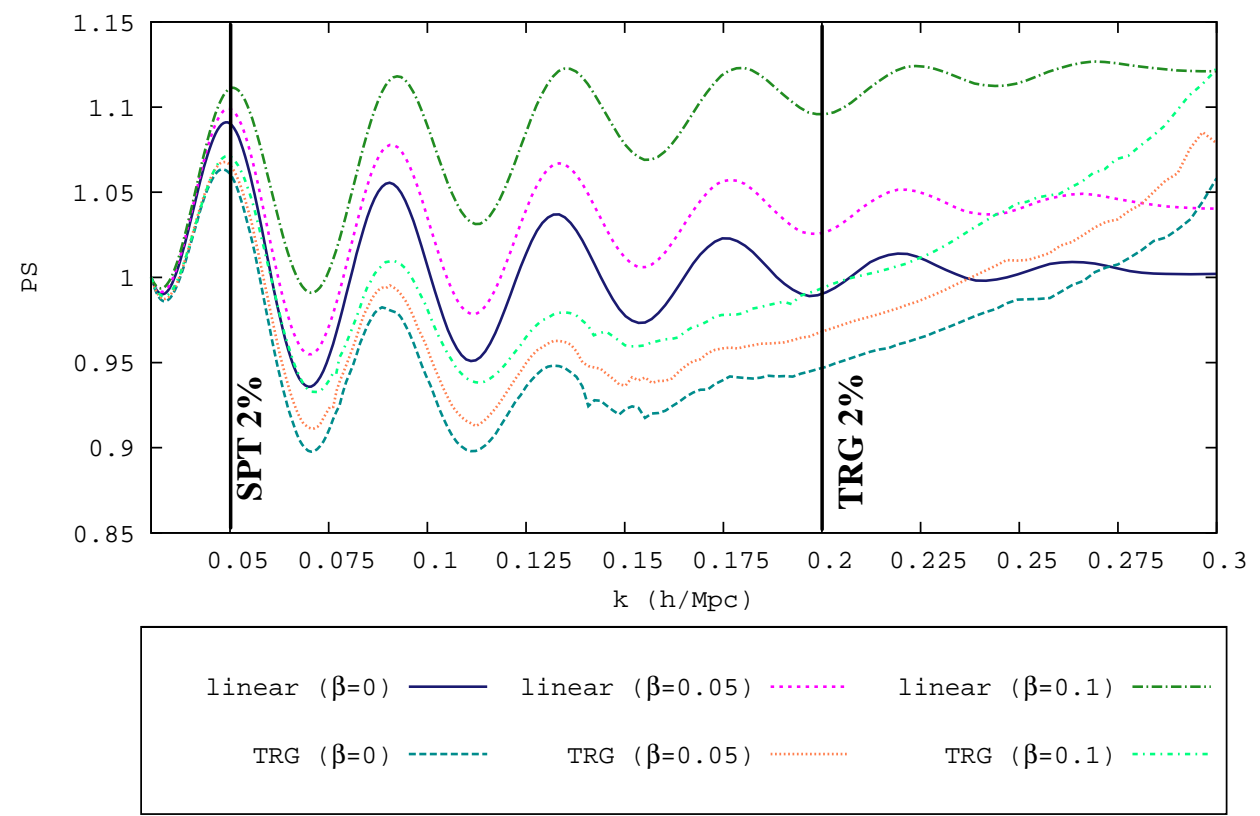

Figure 5: Dark matter velocity-velocity spectra for various $\beta$ at $z=0$,normalized similarly to those in fig. 3 .

40, 45], in which the emphasis is put on the halo region of the spectrum.

\section{Conclusions}

In this paper we have extended the TRG formalism introduced in ref. [5] in two respects. Firstly, we described the matter sector of the theory keeping track of the CDM and BM components separately, instead of treating them as a single fluid. Secondly, we introduced a new scalar force that couples differently to CDM and BM. As was discussed recently in [26], an accurate computation of the evolution of the different components is mandatory if one wants to achieve high precision modeling of structure formation. In order to test the accuracy of the TRG method for this more general class of cosmological scenaria, we analyzed the same cosmologies considered in [40, and compared our results with those of the N-body simulations presented there. The agreement is very good up to $k \simeq 0.5 \mathrm{~h} / \mathrm{Mpc}$ at $z=0$, where the non-linear power spectrum is roughly twice the linear one. These results confirm the reliability of the TRG as a computational tool that can fill the gap between linear and non-linear scales.

Even in the absence of an extra force, or in the case that the extra force couples universally to all matter species, the evolution of BM and CDM differs as a consequence of different initial conditions after decoupling. In linear theory, the initial unbalance between BM and CDM fluctuations is almost completely washed out by the present epoch. However, when non-linearities are taken into account the bias persists. We have seen that the effect is at the percent level in the BAO range at low redshift in the uncoupled case, but it may grow up to the $2.5 \%$ level when a non-zero coupling is turned on with a value compatible with present bounds (obtained within the linear approximation [38, 39]). As a result, these models will receive significant constraints from future galaxy surveys, which aim to measure the power spectrum within the BAO range with an accuracy at the percent level. A thorough investigation of this model-dependent issue goes beyond the purpose of this paper and is postponed for future work.

The most interesting outcome of our analysis from the point of view of observations concerns 


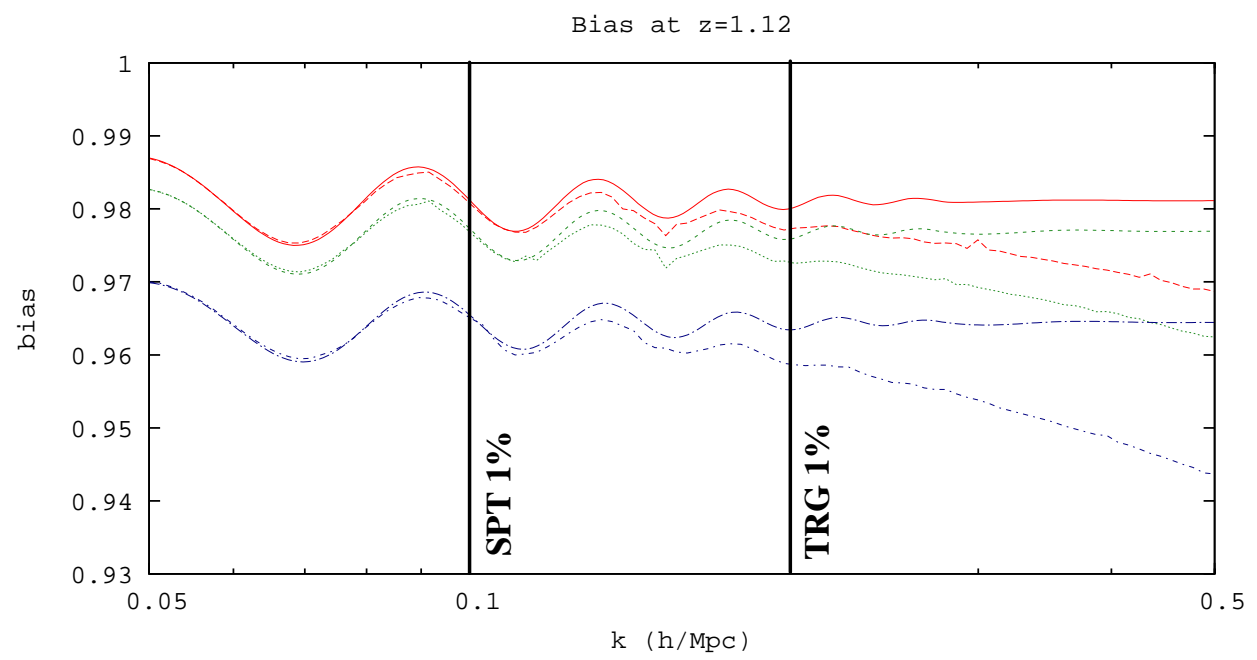

$$
\begin{aligned}
& \beta=0 \text {, linear } \quad \beta=0.05 \text {, linear ......... } \beta=0.1 \text {, linear } \ldots \\
& \beta=0 \text {, TRG - } \quad \beta=0.05, \text { TRG } \ldots \ldots \ldots \ldots \ldots
\end{aligned}
$$

Figure 6: The bias in the BAO region for $z=1.12$.

the form of the bias between CDM and BM spectra. In linear theory, the bias is scale independent in recent epochs [25. The non-linear corrections remove this feature, and make the bias scale dependent, consistently with the results of [40]. We confirm and extend these results for the BAO region $(0.03 h / \mathrm{Mpc} \lesssim k \lesssim 0.25 h / \mathrm{Mpc})$, where we expect the TRG method to be reliable. The effect becomes more pronounced with increasing coupling between CDM and DE (assuming that the respective coupling for BM vanishes). The form of the bias provides an additional observational handle for the differentiation between various models. For example, in models with massive neutrinos the growth of the spectrum induced by the non-linearities at small length scales is compensated by the free-streaming of neutrinos 6. On the other hand, the bias is expected to remain scale-dependent at the non-linear level if there is a substantial CDM-DE coupling.

\section{Acknowledgments}

F. S. would like to thank C. Nardini and D. Seminara for useful discussions. We are grateful to M. Baldi for providing the N-Body data of [0] for comparison with our results. M. P. and N. T. are supported in part by the EU Marie Curie Network "UniverseNet" (MRTN-CT-2006-035863). $\mathrm{N}$. T is also supported in part by the ITN network "UNILHC" (PITN-GA-2009-237920). V. P. acknowledges support from the Alexander von Humboldt Foundation. G. R. is supported by the German Aeronautics Center and Space Agency (DLR), under program 50-OP-0901, funded by the Federal Ministry of Economics and Technology.

\section{Appendix A: Non-linear evolution of perturbations}

We consider first the possibility that the energy density of the Universe is dominated by two components: a) a species of weakly interacting, massive particles, which we identify with dark matter $(\mathrm{CDM})$, and b) a slowly varying, classical scalar field $\phi$, whose contribution is characterized as dark energy (DE). There is a direct coupling between the particles and the scalar field: The mass $m$ of the 
particles depends on the value of $\phi$. For classical particles, the action of the system can be written as

$$
\mathcal{S}=\int d^{4} x \sqrt{-g}\left(\frac{1}{16 \pi G} R+\frac{1}{2} \frac{\partial \phi}{\partial x^{\mu}} \frac{\partial \phi}{\partial x^{\nu}} g^{\mu \nu}-U(\phi)\right)-\sum_{i} \int m\left(\phi\left(x_{i}\right)\right) d s_{i},
$$

with $d s_{i}=\sqrt{g_{\mu \nu}\left(x_{i}\right) d x_{i}^{\mu} d x_{i}^{\nu}}$ and the second integral taken over particle trajectories. Variation of the action with respect to $\phi$ results in the equation of motion

$$
\begin{aligned}
\frac{1}{\sqrt{-g}} \frac{\partial}{\partial x^{\mu}}\left(\sqrt{-g} g^{\mu \nu} \frac{\partial \phi}{\partial x^{\nu}}\right) & =-\frac{d U}{d \phi}-\frac{1}{\sqrt{-g}} \sum_{i} \int d s_{i} \frac{d m\left(\phi\left(x_{i}\right)\right)}{d \phi} \delta^{(4)}\left(x-x_{i}\right) \\
& =-\frac{d U}{d \phi}+\frac{\beta(\phi)}{M}\left(T_{C D M}\right)_{\mu}^{\mu},
\end{aligned}
$$

with the energy-momentum tensor associated with the gas of particles given by

$$
\left(T_{C D M}\right)^{\mu \nu}=\frac{1}{\sqrt{-g}} \sum_{i} \int d s_{i} m\left(\phi\left(x_{i}\right)\right) \frac{d x_{i}^{\mu}}{d s_{i}} \frac{d x_{i}^{\nu}}{d s_{i}} \delta^{(4)}\left(x-x_{i}\right)
$$

Here $M$ is the reduced Planck mass $M=(8 \pi G)^{-1 / 2}$ and we have defined $\beta(\phi) / M=-d \ln m(\phi) / d \phi$. In the following we shall use eq. (5.2), but we shall approximate the energy-momentum tensor as that of an ideal pressureless fluid. The same equation is obtained for scalar-tensor theories of gravity in the Einstein frame. If more than one massive species are present in such theories, their coupling to the scalar field is universal. In order to be as general as possible when we discuss multiple massive species in the following appendix, we assume that each species has a different coupling $\beta_{i}$, induced through the $\phi$-dependence of its mass.

For the metric, we consider an ansatz of the form (2.2). We assume that the Newtonian potential $\Phi$ is weak, $\Phi \ll 1$, and that the field $\phi$ can be decomposed as in eq. (2.3), with $\delta \phi / \bar{\phi} \ll 1$. In general, $\bar{\phi}=\mathcal{O}(M)$. The magnitude of the fluctuations of $\phi$ is expected to be similar to that of the gravitational field $\Phi$. The reason is that the source for both is the dark matter density, to which they couple with comparable strength. Finally, the density can be decomposed as in eq. (2.4). Our aim is to take into account the effect of the local velocity field $\delta \vec{v}$, when this becomes significant because of large field gradients. For subhorizon perturbations with momenta $k \gg \mathcal{H}=\dot{a} / a$, the linear analysis predicts $\delta \vec{v} \sim(k / \mathcal{H}) \Phi \sim(\mathcal{H} / k)(\delta \rho / \bar{\rho})$. We assume that these relations are approximately valid even at the non-linear level, within the range of validity of our scheme. Our assumptions can be summarized in the hierarchy of scales: $\Phi, \delta \phi / \bar{\phi} \ll|\delta \vec{v}| \ll \delta \rho / \bar{\rho} \lesssim 1$. At the linear level, we have $\delta \vec{v}^{2} \sim \Phi(\delta \rho / \bar{\rho})$. We assume that such a relation holds at the non-linear level as well. As we are dealing with subhorizon perturbations, we expect that the spatial derivatives of $\Phi, \delta \phi$ dominate over their time derivatives. Following linear theory, we make the more specific assumption that a spatial derivative acting on $\Phi, \delta \phi$ or $\delta \vec{v}$ increases the position of that quantity in the hierarchy by one level. In this sense $\vec{\nabla} \Phi$ is comparable to $\delta \vec{v}$, while $\nabla^{2} \Phi$ is comparable to $\bar{\rho}$.

We approximate the energy-momentum tensor of dark matter as $\left(T_{C D M}\right)^{\mu \nu}=\rho V^{\mu} V^{\nu}$. We define the peculiar velocity through $V^{i}=\delta v^{i} / a$. Keeping the leading terms in our expansion gives

$$
\begin{aligned}
& \left(T_{C D M}\right)_{0}^{0}=\rho\left(1+\delta \vec{v}^{2}\right) \\
& \left(T_{C D M}\right)^{0}{ }_{i}=-\rho \delta v^{i} \\
& \left(T_{C D M}\right)^{i}{ }_{j}=-\rho \delta v^{i} \delta v_{j},
\end{aligned}
$$

with $\delta v_{j}=\delta v^{j}$. We emphasize at this point that our assumption for the form of $\left(T_{C D M}\right)^{i j}$ is consistent with the presence of only one gravitational potential $\Phi$ in our ansatz (2.2) for the metric, within the order that this potential will be determined through the Einstein equations.

The energy-momentum tensor of the scalar field has the leading part

$$
\begin{aligned}
\left(T_{S}\right)_{0}^{0} & =\frac{1}{2 a^{2}} \dot{\bar{\phi}}^{2}+U(\bar{\phi}) \\
\left(T_{S}\right)_{i}^{0} & =0 \\
\left(T_{S}\right)_{j}{ }_{j} & =\left[-\frac{1}{2 a^{2}} \dot{\bar{\phi}}^{2}+U(\bar{\phi})\right] \delta_{j}{ }_{j},
\end{aligned}
$$


with a dot denoting a derivative with respect to $\tau$. It also includes a perturbation

$$
\begin{aligned}
\left(\delta T_{S}\right)_{0}^{0} & =\frac{1}{a^{2}}\left[-\dot{\bar{\phi}}^{2} \Phi+\dot{\bar{\phi}} \delta \dot{\phi}+\frac{1}{2}(\vec{\nabla} \delta \phi)^{2}+a^{2} \frac{d U(\bar{\phi})}{d \phi} \delta \phi\right] \\
\left(\delta T_{S}\right)_{i}^{0} & =\frac{1}{a^{2}} \dot{\bar{\phi}} \delta \phi, i \\
\left(\delta T_{S}\right)_{j}{ }_{j} & =\frac{1}{a^{2}}\left[\dot{\bar{\phi}}^{2} \Phi-\dot{\bar{\phi}} \delta \dot{\phi}+\frac{1}{2}(\vec{\nabla} \delta \phi)^{2}+a^{2} \frac{d U(\bar{\phi})}{d \phi} \delta \phi\right] \delta_{j}{ }_{j}-\frac{\partial^{i}(\delta \phi) \partial_{j}(\delta \phi)}{a^{2}} .
\end{aligned}
$$

Notice that we have included a term $(\vec{\nabla} \delta \phi)^{2} / 2$, as it is comparable to $\delta \vec{v}^{2}$ or $\Phi$ within our assumed hierarchy. The same is not true for $(\delta \dot{\phi})^{2} / 2$, which is subleading.

The equation of motion of the scalar field (5.2) can be split into an equation for the homogeneous part:

$$
\ddot{\bar{\phi}}+2 \frac{\dot{a}}{a} \dot{\bar{\phi}}+a^{2} \frac{d U(\bar{\phi})}{d \phi}=+\frac{\beta(\bar{\phi})}{M} a^{2} \bar{\rho}
$$

and one for the perturbation:

$$
\delta \ddot{\phi}+2 \frac{\dot{a}}{a} \delta \dot{\phi}-\nabla^{2} \delta \phi+a^{2} \frac{d^{2} U(\bar{\phi})}{d \phi^{2}} \delta \phi-4 \dot{\bar{\phi}} \dot{\Phi}+2 a^{2} \frac{d U(\bar{\phi})}{d \phi} \Phi=\frac{\beta(\bar{\phi})}{M} a^{2} \delta \rho+\frac{1}{M} \frac{d \beta(\bar{\phi})}{d \phi} a^{2}(\bar{\rho}+\delta \rho) \delta \phi
$$

The terms with time derivatives are subdominant according to our assumptions. Neglecting them results in

$$
-\nabla^{2} \delta \phi+\left[a^{2} \frac{d^{2} U(\bar{\phi})}{d \phi^{2}}-\frac{1}{M} \frac{d \beta(\bar{\phi})}{d \phi}(\bar{\rho}+\delta \rho)\right] \delta \phi+2 a^{2} \frac{d U(\bar{\phi})}{d \phi} \Phi=\frac{\beta(\bar{\phi})}{M} a^{2} \delta \rho .
$$

For the scalar field $\bar{\phi}$ to evolve at cosmological times, it must have a mass term $d^{2} U(\bar{\phi}) / d \phi^{2}=\mathcal{O}\left(\mathcal{H}^{2}\right)$. We also have $U(\bar{\phi}), \bar{\rho}=\mathcal{O}\left(\mathcal{H}^{2} M^{2}\right)$. It is natural to expect $d U(\bar{\phi}) / d \phi=\mathcal{O}\left(\mathcal{H}^{2} M\right)$. For subhorizon perturbations with momenta $k \gg \mathcal{H}$, neglecting the subleading terms results in a very simple Poisson equation for the field $\delta \phi$ :

$$
\nabla^{2} \delta \phi=-\frac{\beta(\bar{\phi})}{M} a^{2} \delta \rho
$$

The equation of motion for the gravitational potential $\Phi$ can be obtained from the first Einstein equation. The leading terms give

$$
\mathcal{H}^{2}=\left(\frac{\dot{a}}{a}\right)^{2}=\frac{1}{3 M^{2}}\left[a^{2} \bar{\rho}+\frac{1}{2} \dot{\bar{\phi}}^{2}+a^{2} U(\bar{\phi})\right],
$$

while the equation for the perturbation is

$$
\nabla^{2} \Phi-3 \mathcal{H} \dot{\Phi}-3 \mathcal{H}^{2} \Phi=\frac{1}{2 M^{2}}\left[a^{2} \delta \rho+a^{2} \bar{\rho} \delta \vec{v}^{2}-\dot{\bar{\phi}}^{2} \Phi+\dot{\bar{\phi}} \delta \dot{\phi}+a^{2} \frac{d U(\bar{\phi})}{d \phi} \delta \phi\right] .
$$

Our assumptions about the hierarchy of the various scales and the dominance of the spatial derivatives lead to the Poisson equation for the gravitational field $\Phi$ :

$$
\nabla^{2} \Phi=\frac{1}{2 M^{2}} a^{2} \delta \rho
$$

We now turn to equations derived from the conservation of the total energy-momentum tensor $T_{; \nu}^{\mu \nu}=0$. For $\mu=0$, the leading terms give

$$
\dot{\bar{\rho}}+3 \mathcal{H} \bar{\rho}=-\frac{\dot{\bar{\phi}}}{a^{2}}\left(\ddot{\bar{\phi}}+2 \mathcal{H} \dot{\bar{\phi}}+a^{2} \frac{d U(\bar{\phi})}{d \phi}\right)=-\frac{\beta(\bar{\phi})}{M} \dot{\bar{\phi}} \bar{\rho},
$$

where we have employed eq. (5.7). The equation for the perturbations is more complicated. It can be simplified considerably through our assumptions about the hierarchy of the various fields. We obtain

$$
\delta \dot{\rho}+3 \mathcal{H} \delta \rho+\vec{\nabla}[(\bar{\rho}+\delta \rho) \delta \vec{v}]=\frac{\dot{\bar{\phi}}}{a^{2}} \nabla^{2} \delta \phi=-\frac{\beta(\bar{\phi})}{M} \dot{\bar{\phi}} \delta \rho,
$$


where we have employed eq. (5.10).

For $\mu=i=1,2,3$ we obtain the generalization of the Euler equation for this system. After eliminating higher-order terms we find

$$
\begin{aligned}
(\bar{\rho}+\delta \rho)[\delta \dot{\vec{v}}+\mathcal{H} \delta \vec{v}+(\delta \vec{v} \vec{\nabla}) \delta \vec{v}] & +[\dot{\bar{\rho}}+3 \mathcal{H} \bar{\rho}+\delta \dot{\rho}+3 \mathcal{H} \delta \rho+\vec{\nabla}[(\bar{\rho}+\delta \rho) \delta \vec{v}]] \delta \vec{v} \\
=-(\bar{\rho}+\delta \rho) \vec{\nabla} \Phi & +\frac{1}{a^{2}}\left(\ddot{\bar{\phi}}+2 \mathcal{H} \dot{\bar{\phi}}-\nabla^{2} \delta \phi+a^{2} \frac{d U(\bar{\phi})}{d \phi}\right) \vec{\nabla} \delta \phi .
\end{aligned}
$$

Employing eqs. (5.7), (5.10), (5.14), (5.15) we find

$$
\delta \dot{\vec{v}}+\left(\mathcal{H}-\frac{\beta(\bar{\phi})}{M} \dot{\bar{\phi}}\right) \delta \vec{v}+(\delta \vec{v} \vec{\nabla}) \delta \vec{v}=-\vec{\nabla} \Phi+\frac{\beta(\bar{\phi})}{M} \vec{\nabla} \delta \phi
$$

For constant $\beta$, we can form a combination of eqs. (5.10), (5.13) of the form

$$
\nabla^{2} \tilde{\Phi}=\frac{1}{2 \tilde{M}} a^{2} \delta \rho
$$

where $\tilde{\Phi}=\Phi-\beta \delta \phi / M$ and $\tilde{M}^{2}=M^{2} /\left(1+2 \beta^{2}\right)$. Eq. (5.17) becomes

$$
\delta \dot{\vec{v}}+\left(\mathcal{H}-\frac{\beta}{M} \dot{\bar{\phi}}\right) \delta \vec{v}+(\delta \vec{v} \cdot \vec{\nabla}) \delta \vec{v}=-\vec{\nabla} \tilde{\Phi}
$$

We can see that the Newtonian potential for the perturbations involves a stronger Newton's constant $\tilde{G}=\left(8 \pi \tilde{M}^{2}\right)^{-1}=\left(1+2 \beta^{2}\right) G$. There is also a correction $\sim \dot{\bar{\phi}} \delta \vec{v}$ in the l.h.s. of the Euler equation, because the particles do not follow geodesic motion with respect to the background metric.

\section{Appendix B: Several species of non-relativistic matter}

In this appendix we generalize the formalism to the case that there are more than one particle species contributing significantly to the energy density. Each of them couples to the scalar field with a different coupling $\beta_{i}$. One example of particular interest includes CDM with $\beta_{C D M} \neq 0$ and baryonic matter (BM), for which we assume that $\beta_{B M}=0$ in order to be consistent with observational constraints. We also normalize all dimensionful quantities in terms of the Planck mass. This is equivalent to setting $M=1$ in the expressions of appendix A.

The background equations are

$$
\begin{aligned}
\mathcal{H}^{2} & =\frac{1}{3}\left[a^{2} \sum_{i} \bar{\rho}_{i}+\frac{1}{2} \dot{\bar{\phi}}^{2}+a^{2} U(\bar{\phi})\right] \\
\dot{\bar{\rho}}_{i}+3 \mathcal{H} \bar{\rho}_{i} & =-\dot{\bar{\phi}} \beta_{i} \bar{\rho}_{i} . \\
\ddot{\bar{\phi}}+2 \mathcal{H} \dot{\bar{\phi}}+a^{2} \frac{d U(\bar{\phi})}{d \phi} & =a^{2} \sum_{i} \beta_{i} \bar{\rho}_{i},
\end{aligned}
$$

with the index $i$ counting the various species.

For the perturbations, eq. (5.10) becomes

$$
\nabla^{2} \delta \phi=-a^{2} \sum_{i} \beta_{i} \delta \rho_{i}
$$

and eq. (5.13)

$$
\nabla^{2} \Phi=\frac{1}{2} a^{2} \sum_{i} \delta \rho_{i}
$$

Eq. (5.15) generalizes to

$$
\delta \dot{\rho}_{i}+3 \mathcal{H} \delta \rho_{i}+\vec{\nabla}\left[\left(\bar{\rho}_{i}+\delta \rho_{i}\right) \delta \vec{v}_{i}\right]=-\beta_{i} \dot{\bar{\phi}} \delta \rho_{i} .
$$


Finally, eq. (5.17) becomes

$$
\delta \dot{\vec{v}}_{i}+\left(\mathcal{H}-\beta_{i} \dot{\bar{\phi}}\right) \delta \vec{v}_{i}+\left(\delta \vec{v}_{i} \cdot \vec{\nabla}\right) \delta \vec{v}_{i}=-\vec{\nabla} \Phi+\beta_{i} \vec{\nabla} \delta \phi .
$$

We can write the above equations in a more useful form by defining the density contrasts $\delta_{i} \equiv$ $\delta \rho_{i} / \bar{\rho}_{i} \lesssim 1$ and $\theta_{i}(\mathbf{k}, \tau) \equiv \vec{\nabla} \cdot \delta \vec{v}_{i}(\mathbf{k}, \tau)$. For the density contrasts we obtain

$$
\dot{\delta}_{i}+\vec{\nabla}\left[\left(1+\delta_{i}\right) \overrightarrow{\delta v}_{i}\right]=0 .
$$

For the Fourier transformed quantities, eq. (6.8) gives eq. (2.8), while eq. (2.7b) gives eq. (2.10).

We replace time by the variable $\eta=\ln a(\tau)$. For $n$ species of non-relativistic matter we define the field $\varphi(\mathbf{k}, \eta)$ as a vector with $2 n$ components:

$$
\varphi(\mathbf{k}, \eta)=\left(\begin{array}{c}
\varphi_{1}(\mathbf{k}, \eta) \\
\varphi_{2}(\mathbf{k}, \eta) \\
\vdots \\
\varphi_{2 n-1}(\mathbf{k}, \eta) \\
\varphi_{2 n}(\mathbf{k}, \eta)
\end{array}\right)=e^{-\eta}\left(\begin{array}{c}
\delta_{1}(\mathbf{k}, \eta) \\
-\frac{\theta_{1}(\mathbf{k}, \eta)}{\mathcal{H}} \\
\vdots \\
\delta_{n}(\mathbf{k}, \eta) \\
-\frac{\theta_{n}(\mathbf{k}, \eta)}{\mathcal{H}}
\end{array}\right) .
$$

This allows us to bring eqs. (2.8), (2.10) in the usual form [1, 4, 5]:

$$
\partial_{\eta} \varphi_{a}(\mathbf{k}, \eta)+\Omega_{a b} \varphi_{b}(\mathbf{k}, \eta)=e^{\eta} \gamma_{a b c}\left(\mathbf{k},-\mathbf{k}_{1},-\mathbf{k}_{2}\right) \varphi_{a}\left(\mathbf{k}_{1}, \eta\right) \varphi_{b}\left(\mathbf{k}_{2}, \eta\right) .
$$

The indices $a, b, c$ take values $1, \ldots, 2 n$. Repeated momenta are integrated over while repeated indices are summed over. The functions $\gamma$, that determine effective vertices, are analogous to those employed in [4, 5. We find that the non-zero functions are

$$
\begin{aligned}
\gamma_{2 i-1,2 i, 2 i-1}\left(\mathbf{k}, \mathbf{k}_{1}, \mathbf{k}_{2}\right) & =\frac{\tilde{\alpha}\left(\mathbf{k}_{1}, \mathbf{k}_{2}\right)}{2} \delta_{D}\left(\mathbf{k}+\mathbf{k}_{1}+\mathbf{k}_{2}\right)=\gamma_{2 i-1,2 i-1,2 i}\left(\mathbf{k}, \mathbf{k}_{2}, \mathbf{k}_{1}\right) \\
\gamma_{2 i, 2 i, 2 i}\left(\mathbf{k}, \mathbf{k}_{1}, \mathbf{k}_{2}\right) & =\tilde{\beta}\left(\mathbf{k}_{1}, \mathbf{k}_{2}\right) \delta_{D}\left(\mathbf{k}+\mathbf{k}_{1}+\mathbf{k}_{2}\right) .
\end{aligned}
$$

The $\Omega$-matrix of eq. (6.9) is a $2 n \times 2 n$ matrix. Let us define the $2 \times 2$ matrices $\omega_{i}(\eta)$ and $\omega_{i, j}(\eta)$, with $i, j=1, \ldots, n$ and $i \neq j$, as

$$
\omega_{i}(\eta)=\left(\begin{array}{cc}
1 & -1 \\
-\frac{3}{2} \Omega_{i}\left(2 \beta_{i}^{2}+1\right) & 2-\beta_{i} \bar{\phi}^{\prime}+\frac{\mathcal{H}^{\prime}}{\mathcal{H}}
\end{array}\right)
$$

and

$$
\omega_{i, j}(\eta)=\left(\begin{array}{cc}
0 & 0 \\
-\frac{3}{2} \Omega_{j}\left(2 \beta_{i} \beta_{j}+1\right) & 0
\end{array}\right),
$$

where a prime denotes a derivative with respect to $\eta$. Then, the $\Omega$-matrix can be written as

$$
\Omega(\eta)=\left(\begin{array}{cccc}
\omega_{1} & \omega_{1,2} & \ldots & \omega_{1, n} \\
\omega_{2,1} & \omega_{2} & \ldots & \omega_{2, n} \\
\vdots & & \ddots & \\
& & & \\
\omega_{n, 1} & \omega_{n, 2} & \ldots & \omega_{n}
\end{array}\right) .
$$

Notice that the only way in which different species of matter influence each other is through the matrices $\omega_{i, j}$, while the vertices do not mix contributions from different species. 


\section{References}

[1] M. Crocce and R. Scoccimarro, Phys. Rev. D 73 (2006) 063519 arXiv:astro-ph/0509418.

[2] M. Crocce and R. Scoccimarro, Phys. Rev. D 73 (2006) 063520 arXiv:astro-ph/0509419; Phys. Rev. D 77 (2008) 023533 arXiv:0704.2783 [astro-ph]].

[3] A. Taruya and T. Hiramatsu, arXiv:0708.1367 [astro-ph]; T. Hiramatsu and A. Taruya, Phys. Rev. D 79 (2009) 103526 arXiv:0902.3772 [astro-ph.CO]]; A. Taruya, T. Nishimichi, S. Saito and T. Hiramatsu, arXiv:0906.0507 [astro-ph.CO].

[4] S. Matarrese and M. Pietroni, JCAP 0706 (2007) 026 arXiv:astro-ph/0703563.

[5] M. Pietroni, JCAP 0810 (2008) 036 arXiv:0806.0971 [astro-ph]].

[6] J. Lesgourgues, S. Matarrese, M. Pietroni and A. Riotto, JCAP 0906 (2009) 017 arXiv:0901.4550 [astro-ph.CO]].

[7] P. McDonald, Phys. Rev. D 75 (2007) 043514 arXiv:astro-ph/0606028.

[8] P. Valageas, Astron. Astrophys. 379 (2001) 8 arXiv:astro-ph/0107015; Astron. Astrophys. 382 (2002) 412 arXiv:astro-ph/0107126; Astron. Astrophys. 421 (2004) 23 arXiv:astro-ph/0307008; Astron. Astrophys. 465 (2007) 725 arXiv:astro-ph/0611849.

[9] T. Matsubara, Phys. Rev. D 77 (2008) 063530 arXiv:0711.2521 [astro-ph]]; Phys. Rev. D 78 (2008) 083519 [Erratum-ibid. D 78 (2008) 109901] arXiv:0807.1733 [astro-ph]].

[10] J. Carlson, M. White and N. Padmanabhan, Phys. Rev. D 80 (2009) 043531 arXiv:0905.0479 [astro-ph.CO]].

[11] J. Berges, N. Tetradis and C. Wetterich, Phys. Rept. 363 (2002) 223 arXiv:hep-ph/0005122.

[12] C. Wetterich, Astron. Astrophys. 301 (1995) 321 arXiv:hep-th/9408025.

[13] G. W. Anderson and S. M. Carroll, arXiv:astro-ph/9711288

[14] L. Amendola, Phys. Rev. D 62 (2000) 043511 arXiv:astro-ph/9908023.

[15] G. Mangano, G. Miele and V. Pettorino, Mod. Phys. Lett. A 18 (2003) 831 arXiv:astro-ph/0212518.

[16] G. R. Farrar and P. J. E. Peebles, Astrophys. J. 604 (2004) 1 arXiv:astro-ph/0307316.

[17] R. Mainini and S. A. Bonometto, Phys. Rev. Lett. 93 (2004) 121301 arXiv:astro-ph/0406114;

[18] R. Catena, M. Pietroni and L. Scarabello, Phys. Rev. D $\mathbf{7 0}$ (2004) 103526 arXiv:astro-ph/0407646.

[19] M. Pietroni, Phys. Rev. D 72 (2005) 043535 arXiv:astro-ph/0505615.

[20] V. Pettorino and C. Baccigalupi, Phys. Rev. D 77 (2008) 103003 arXiv:0802.1086 [astro-ph]].

[21] L. Amendola, Phys. Rev. D 69 (2004) 103524 arXiv:astro-ph/0311175.

[22] F. Perrotta, S. Matarrese, M. Pietroni and C. Schimd, Phys. Rev. D 69 (2004) 084004 arXiv:astro-ph/0310359.

[23] B. Bertotti, L. Iess and P. Tortora, Nature 425, 374 (2003).

[24] B. A. Gradwohl and J. A. Frieman, Astrophys. J. 398 (1992) 407.

[25] L. Amendola and D. Tocchini-Valentini, Phys. Rev. D $66 \quad$ (2002) 043528 arXiv:astro-ph/0111535.

[26] G. Somogyi and R. E. Smith, arXiv:0910.5220 [astro-ph.CO].

[27] A. V. Maccio, C. Quercellini, R. Mainini, L. Amendola and S. A. Bonometto, Phys. Rev. D 69 (2004) 123516 arXiv:astro-ph/0309671.

[28] A. Nusser, S. S. Gubser and P. J. E. Peebles, Phys. Rev. D 71 (2005) 083505 arXiv:astro-ph/0412586.

[29] L. Amendola and C. Quercellini, Phys. Rev. D 68 (2003) 023514 arXiv:astro-ph/0303228.

[30] S. Lee, G. C. Liu and K. W. Ng, Phys. Rev. D 73 (2006) 083516 arXiv:astro-ph/0601333. 
[31] B. Wang, J. Zang, C. Y. Lin, E. Abdalla and S. Micheletti, Nucl. Phys. B 778 (2007) 69 arXiv:astro-ph/0607126.

[32] Z. K. Guo, N. Ohta and S. Tsujikawa, Phys. Rev. D 76 (2007) 023508 arXiv:astro-ph/0702015.

[33] R. Mainini and S. Bonometto, JCAP 0706 (2007) 020 arXiv:astro-ph/0703303.

[34] O. Bertolami, F. Gil Pedro and M. Le Delliou, Phys. Lett. B 654 (2007) 165 arXiv:astro-ph/0703462.

[35] I. P. Neupane and H. Trowland, arXiv:0902.1532 [gr-qc].

[36] S. Lee, G. c. Liu and K. w. Ng, arXiv:0910.2175.

[37] W. A. Hellwing and R. Juszkiewicz, arXiv:0809.1976 [astro-ph].

[38] R. Bean, E. E. Flanagan, I. Laszlo and M. Trodden, Phys. Rev. D 78 (2008) 123514 arXiv:0808.1105 [astro-ph]].

[39] G. La Vacca, J. R. Kristiansen, L. P. L. Colombo, R. Mainini and S. A. Bonometto, JCAP 0904 (2009) 007 arXiv:0902.2711 [astro-ph.CO]].

[40] M. Baldi, V. Pettorino, G. Robbers and V. Springel, arXiv:0812.3901 [astro-ph].

[41] N. Brouzakis and N. Tetradis, JCAP 0601 (2006) 004 arXiv:astro-ph/0509755.

[42] F. Bernardeau, S. Colombi, E. Gaztanaga and R. Scoccimarro, Phys. Rept. 367 (2002) 1 arXiv:astro-ph/0112551.

[43] M. Doran, JCAP 0510 (2005) 011 arXiv:astro-ph/0302138.

[44] D. J. Eisenstein and W. Hu, Astrophys. J. 511 (1997) 5 arXiv:astro-ph/9710252.

[45] R. Mainini, Phys. Rev. D 72 (2005) 083514 arXiv:astro-ph/0509318; R. Mainini and S. Bonometto, Phys. Rev. D 74 (2006) 043504 arXiv:astro-ph/0605621]. 